\title{
In Vitro Activity of Rifampicin Combined with Daptomycin or Tigecycline on Staphylococcus haemolyticus Biofilms
}

\author{
Ewa Szczuka $^{1} \cdot$ Katarzyna Grabska $^{1} \cdot$ Adam Kaznowski $^{1}$
}

Received: 4 December 2014/ Accepted: 5 March 2015/Published online: 18 April 2015

(c) The Author(s) 2015. This article is published with open access at Springerlink.com

\begin{abstract}
Staphylococcus haemolyticus is of increasing concern as a cause of several biofilm-associated infections, and today, it represents the second most common organism among clinical isolates of coagulase-negative staphylococci. However, little is known regarding the treatment of infections caused by these bacteria. In this study, we characterize the biofilm formed by S. haemolyticus strains isolated from bloodstream infections and assess in vitro the activity of rifampicin combined with daptomycin or tigecycline against bacteria growing in a biofilm. The results of our studies indicated that the majority (78\%) of methicillin-resistant Staphylococcus haemolyticus strains have the ability to form a biofilm in vitro. None of these strains carried icaADBC genes indicating that they form biofilm via ica-independent mechanisms. The molecular characterization of the biofilm showed that proteins are the predominant matrix component and play a major role in biofilm structure. Extracellular DNA and polysaccharides, other than polysaccharide intercellular adhesin, are also present in the biofilm matrix, but they play a minor role. The images obtained by confocal laser scanning microscopy showed that most S. haemolyticus strains formed a dense biofilm with a low number of dead cells. In vitro study demonstrated excellent activity of tigecycline in combination with rifampicin against cell growth in the proteinous biofilm. The BIC (biofilm inhibitory concentration) value for tigecycline/rifampicin ranged from 0.062 to $1 \mu \mathrm{g} /$ $\mathrm{ml}$, whereas for daptomycin/rifampicin from 0.125 to $2 \mu \mathrm{g} /$ $\mathrm{ml}$. These results indicated that the tigecycline/rifampicin
\end{abstract}

Ewa Szczuka

ewasz@amu.edu.pl

1 Department of Microbiology, Faculty of Biology, Institute of Experimental Biology, Adam Mickiewicz University, ul. Umultowska 89, 61-614 Poznan, Poland combination was more effective against ica-independent biofilm, formed by $S$. haemolyticus strains, than the daptomycin/rifampicin combination.

\section{Introduction}

Staphylococcus haemolyticus belongs to a group of coagulase-negative staphylococci (CoNS) and is a skin commensal, as well as a significant pathogen, with the potential to cause persistent infections, including native valve endocarditis, prosthetic join infections, meningitis, skin and soft tissue infections as well as catheter-related bloodstream infections $[5,9,16,20]$. Currently, S. haemolyticus is ranked as the second highest species of coagulase-negative staphylococci in importance among clinical isolates. This bacterium has the ability to induce apoptosis within host macrophages and also exhibits a cytotoxic effect towards murine macrophages cell lines J774 [10]. However, the virulence of CoNS is mainly attributed to the colonization of an inert, or living surface and biofilm formation. The biofilm represents an adherent, structured community of cells that is embedded in a self-produced, polymeric matrix [22]. The polysaccharide intercellular adhesin (PIA) is a linear homoglycan composed of at least 130 residues of $\beta$-1,6-linked $N$-acetylglucosamine as the major component of the matrix, and plays a crucial role in biofilm formation by S. aureus and S. epidermidis. PIA is the product of $i c a A D B C$ gene expression. Other components, such as cell-wall associated proteins, extracellular DNA, teichoic acid and polysaccharide other than PIA, are capable of mediating intercellular accumulation and biofilm formation by staphylococci [22]. The biofilm growth mode of the bacteria protects them from the host's immune system and antibiotics. Thus, biofilm-associated infections become 
chronic and their eradication by antibiotic therapy is quite rare [13, 21]. In addition, S. haemolyticus is frequently resistant to multiple antibiotics, including $\beta$-lactams, and in such cases, only a few therapeutic alternatives are available. Glycopeptides, vancomycin and teicoplanin have been recommended as a first-line therapy for the treatment of methicillin-resistant staphylococci infections. Moreover, many reports described $S$. haemolyticus strains with a reduced susceptibility to teicoplanin $[1,26]$. The disadvantage of vancomycin is its lack of activity against organisms growing in biofilm and poor tissue and intracellular penetration. Rifampicin has been shown to be one of the most potent in the eradication of staphylococci biofilm. However, rifampicin should not be used as a single agent to treat staphylococci infection, due to the rapid development of resistance [4]. Therefore, extensive studies regarding the use of the rifampicin, in combination with other antibiotics, should be considered in the treatment biofilm-associated infections. A recent study indicated that tigecycline, a glycylcycline antibiotic, exhibits excellent activity in vitro and in vivo against S. aureus strains [3]. Also, daptomycin, a novel cyclic lipopeptide antibiotic, with a unique mechanism of action against Gram-positive bacteria, and the low occurrence of spontaneous resistance against this antibacterial factor, has been successfully used for the treatment of bacteraemia caused by $S$. aureus. Furthermore, this antibiotic also demonstrated the ability to successfully penetrate into $S$. epidermidis biofilm [12, 23].

The purpose of this study was to characterize the biofilm formed by $S$. haemolyticus strains and assess in vitro the activity of rifampicin, in combination with daptomycin, and with tigecycline, against bacteria growing in a biofilm.

\section{Materials and Methods}

\section{Bacterial Strains}

Sixty-five S. haemolyticus strains were isolated from blood taken from in-patients at a hospital in Poznań, Poland. Only one isolate from one patient with bloodstream infections was included. Identification was performed using a Vitek 2 system (bioMérieux, France). All the isolates included in our studies were resistant to oxacillin. The resistance to methicillin was confirmed by the presence of the mecA gene, using the PCR method [7].

\section{Detection of the ica Operon}

Chromosomal DNA was extracted using the Genomic DNA prep Plus kit (A\&A Biotechnology, Gdynia, Poland). The PCR technique was applied to detect the presence of icaADBC genes in $S$. haemolyticus strains as described by
Chokr et al. [2]. Amplification products were electrophoresed in $1.5 \%$ agarose gel, stained with ethidium bromide and documented with a V.99 Bio-Print system (Vilber Lourmat, Torcy, France).

\section{Determination of Biofilm Production by Microtiter Plate Assay}

To test the isolates for biofilm formation, a quantitative adherence assay was used [6]. Briefly, the overnight culture grown in a tryptic soy broth (TSB, Difco, Beckton Dickinson, France) supplemented with $0.25 \%$ glucose was diluted 1:100 in $\mathrm{TSB}_{\text {glucose } 0,25 \%}$ and $100 \mu \mathrm{l}$ was transferred into 96 -well polystyrene microtiter plates. The plates were incubated overnight at $37{ }^{\circ} \mathrm{C}$, then the medium was removed from each of the wells and the plates were gently washed three times, with phosphate-buffered saline (PBS) to remove non-adherent cells. Then, the biofilms in the wells were stained with a $0.4 \%$ crystal violet solution. After washing, the crystal violet from the biofilm was dissolved using an ethanol-acetone mixture (70:30) and absorbance was determined. The strains were considered as biofilm positive if they had an $\mathrm{OD}_{490}>0.250$. Each isolate was tested in triplicate.

\section{Biofilm Detachment Assay}

The biofilm in 96-well microtiter plates was rinsed three times with PBS and treated with $40 \mathrm{mM} \mathrm{NaIO}{ }_{4}$ in $\mathrm{H}_{2} \mathrm{O}$, or $0.1 \mathrm{mg} / \mathrm{ml}$ of proteinase $\mathrm{K}$ in $20 \mathrm{mM}$ Tris- $\mathrm{HCl}(\mathrm{pH} 7.5)$ with $100 \mathrm{mM} \mathrm{NaCl}$, or $0.5 \mathrm{mg} / \mathrm{ml} \mathrm{DNase} \mathrm{(Sigma)} \mathrm{in}$ $5 \mathrm{mM} \mathrm{MgCl}{ }_{2}$. After 24 -h at $37^{\circ} \mathrm{C}$, the biofilms were stained with crystal violet as described above. The percentage of detachment was calculated on the basis of the average difference between the wells subjected to $\mathrm{NaIO}_{4}$, proteinase $\mathrm{K}$, DNase and the control wells untreated with these factors. The detachment results were divided into three categories: no detachment $(<10 \%)$, intermediate detachment (10-50\%) and strong detachment $(>50 \%)$ [6].

\section{Confocal Laser Scanning Microscopy (CLSM)}

Overnight cultures of various strains were inoculated into a coverglass cell-culture chamber (Nunc) [18]. After 24-h incubation at $37{ }^{\circ} \mathrm{C}$, the chamber coverglasses were washed gently three times with PBS to remove planktonic cells. Bacteria in biofilms were stained using SYTO and PI (Live/Dead BacLight Bacterial Viability kits, Invitrogen) for $15 \mathrm{~min}$, and observed under microscope (Carl Zeiss LSM 510- Axiovert 200M) with detector and filter sets for monitoring SYTO9, and PI Carl Zeiss confocal software was used for the analysis of the biofilm, which permitted 
the collection of image stacks and the three-dimensional (3D) visualization of the biofilms.

\section{Biofilm Susceptibility Assay}

Bacterial biofilms were formed on a modified polystyrene microtiter lid (Nunc, TSP system), and the effect of antibiotics was determined according to the procedure described by Moskowitz et al. [15]. Briefly, 22-h-old bacterial cultures of each strain, in TSB with a $1 \%$ glucose medium, were adjusted to a turbidity of $0.5 \mathrm{McF}$ arland, and $100 \mu \mathrm{l}$ of these cultures was transferred to wells of a 96-well microtiter plate covered with a 96-peg lid and incubated for a further 20-h, to allow biofilm development. The peg lids were rinsed three times in sterile water and placed into a new microtiter plate containing two-fold dilutions of antibiotics (e.g. 1024, 512, 256, 128, 64, 32, 16, 8, 4, 2, 1, 0.5, $0.25,0.125,0.062$, and $0.030 \mu \mathrm{g} / \mathrm{mL}$ ) and incubated for $18-20 \mathrm{~h}$ at $37{ }^{\circ} \mathrm{C}$. The combination antibiotic solution contains the two agents in equal concentration. After this time, the biofilm cells were transferred by sonication from the pegs into recovery media, and the optical density at $650 \mathrm{~nm}$ was measured on a plate reader. The plate was incubated for six further hours, and the next bacterial growth was measured by optical density $\left(\mathrm{OD}_{650}\right)$. Biofilm growth was defined as a mean $\mathrm{OD}_{650}$ difference $\left(\mathrm{OD}_{650}\right.$ at $6 \mathrm{~h}$ minus $\mathrm{OD}_{650}$ at $\left.0 \mathrm{~h}\right) \geq 0.05$ for the biofilm control. The BIC values were defined as the lowest concentration without growth.

\section{Results}

Fifty-one (78\%) methicillin-resistant S. haemolyticus strains were able to adhere to polystyrene and to form a biofilm on this surface. None of these strains gave an amplicon in the PCR assays with primers designed for four different regions of icaADBC genes. We estimated the composition of the biofilm matrix, by treating the biofilm with proteinase $\mathrm{K}, \mathrm{NaIO}_{4}$ or DNAse. Firstly, biofilms of $S$. haemolyticus were very effectively dispersed, when proteinase $\mathrm{K}$ was added to a 24-h biofilm. Thirty-six strains showed strong detachment, and only fifteen strains showed intermediate detachment. These results clearly indicate that proteins constitute a structural part of the biofilm matrix. Only one studied isolate showed $>50 \%$ detachment when biofilm was treated with sodium metaperiodate $\left(\mathrm{NaIO}_{4}\right)$, a polysaccharide degrading agent. Many strains showed low detachment $(<30 \%)$ suggesting that polysaccharide may play a minor role in the biofilm structure of S. haemolyticus strains. Our study also indicated that DNAse has an effect on mature S. haemolyticus biofilm. Thirty-five isolates showed more than $25 \%$ detachment of 24-h biofilm, after treatment with DNAse. Although the biofilm matrix was different in chemical composition, proteins were the main component of biofilm, in all tested strains.

The images obtained by CLSM indicate that the $S$. haemolyticus strains formed a complex 3D structure (Fig. 1). Additionally, in the mature biofilm produced by $S$. haemolyticus strains, a low number of dead cells were found. The thickness of the biofilm formed by these strains ranged from 11 to $16 \mu \mathrm{m}$. Apart from the differences in biofilm thickness, we also observed differences in biofilm density and the area of the growth chamber covered by these structures. However, most strains formed a biofilm in which cells were very closely packed.

The 24 strains that created the most condensed biofilm structures were chosen for further study, aiming to estimate the most effective antibiotics in treating the biofilm-associated infection caused by S. haemolyticus strains. When results were interpreted according to EUCAST breakpoints, planktonic forms of these strains were sensitive to rifampicin, tigecycline and daptomycin. The BIC value for tigecycline/rifampicin against cell growth in the biofilm ranged from 0.062 to $1 \mu \mathrm{g} / \mathrm{ml}$, whereas the BIC for daptomycin/rifampicin was 0.125 to $2 \mu \mathrm{g} / \mathrm{ml}$ (Table 1 ). Only two isolates formed a biofilm that exhibited resistance to tigecycline (defined according to EUCAST planktonic susceptibility breakpoints). Sixteen strains of the 24 (67\%) had BIC values $\leq 1 \mu \mathrm{g} / \mathrm{ml}$ and these bacteria are categorized as susceptible to daptomycin, according to planktonic MIC $(\leq 1 \mu \mathrm{g} / \mathrm{ml})$. The results obtained in this study indicate that rifampicin in combination with tigecycline is more active against the bacteria within biofilm than daptomycin/rifampicin. The BIC value of tigecycline in combination with rifampicin was at least two to fivefold higher for 14 strains grown as a biofilm than the daptomycin/ rifampicin. Nonetheless, for seven isolates, tigecycline/rifampicin was only slightly better (i.e. onefold higher) than daptomycin/rifampicin. Furthermore, daptomycin/rifampicin displayed the same effect on biofilms formed by only three isolates as tigecycline/rifampicin.

\section{Discussion}

Our study revealed that the majority of clinical $S$. haemolyticus strains have the ability to form biofilm in vitro. There is evidence that these strains form biofilm via ica-independent mechanisms. The results of this study are similar to previously reported data, which indicated that $i c a D$ genes are present only in three of the 72 tested isolates of S. haemolyticus [6]. According to our knowledge, the components of $S$. haemolyticus biofilm were only previously analysed by Fredheim et al. [6]. In this Norwegian study, DNAse, proteinase $\mathrm{K}$ and NAIO4 caused biofilm 

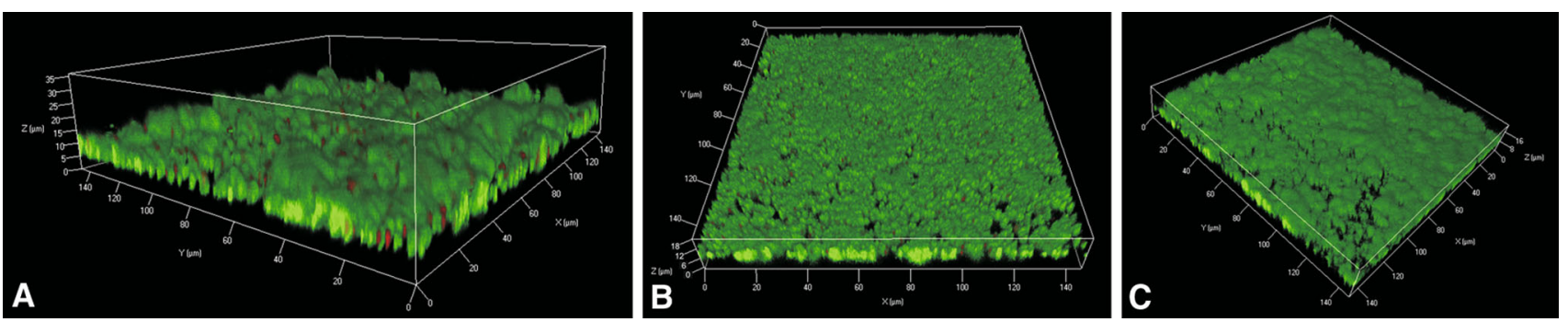

Fig. 1 CLSM images of 24-h biofilm of S. haemolyticus MPU S 12 (a), MPU S 26 (b) and MPU S 54 (c) stained with SYTO9 (green cells) and PI (red cells)

Table 1 In vitro antimicrobial susceptibilities of $S$. haemolyticus biofilm

\begin{tabular}{lll}
\hline BIC $(\mu \mathrm{g} / \mathrm{ml})$ of the following antimicrobial agents & Number of isolates \\
\hline Tigecycline/rifampicin & Daptomycin/rifampicin & \\
\hline 0.062 & 2 & 6 \\
0.062 & 1 & 2 \\
0.062 & 0.5 & 2 \\
0.125 & 1 & 2 \\
0.125 & 0.5 & 2 \\
0.125 & 0.250 & 3 \\
0.125 & 0.125 & 1 \\
0.250 & 0.250 & 2 \\
0.5 & 1 & 2 \\
1 & 2 & 2 \\
\hline
\end{tabular}

detachment for 100, 98 and $38 \%$ of S. haemolyticus isolates, respectively. In our study, proteinase $\mathrm{K}$ caused significant detachment of bacteria in all biofilms, indicating that proteins are the predominant biofilm matrix component and play a major role in biofilm structure. This is in contrast to most other staphylococci species, in which PIA is the essential component of the biofilm matrix and plays a leading role in biofilm development. However, it has also been reported that $S$. epidermidis and $S$. aureus are able to form biofilm via proteinaceous process [22]. According to our study, polysaccharides, different from PIA, are also present in biofilm matrix formed by the majority of $S$. haemolyticus strains, but they play a minor role in biofilm shaping. Additionally, we also found that DNA is present in all biofilms formed by $S$. haemolyticus strains. Besides evaluation of biochemical components of the biofilm matrix, we also analysed $S$. haemolyticus biofilm structures. CLSM observation clearly demonstrated that most $S$. haemolyticus formed dense biofilm structures. These biofilms are not as thick as the PIA-dependent biofilms formed by $S$. aureus and $S$. epidermidis $[24,25]$.

Numerous studies indicate that biofilm-associated infections are extremely difficult to treat $[13,22,23]$. Unfortunately, only a few antimicrobials have the ability to penetrate efficiently staphylococcal biofilm and be efficient against cells in a stationary phase. It should be emphasized that the majority of studies are focused on the PIA-dependent biofilm formed by the $S$. aureus and $S$. epidermidis strains [8, 11, 17, 19, 27]. In our previous studies, we showed that the MIC value for tigecycline against $S$. epidermidis growing in the biofilm ranged from 0.125 to $2 \mu \mathrm{g} / \mathrm{mL}$. Tigecycline in combination with rifampin demonstrated higher activity against bacteria embedded in biofilms than tigecycline alone [24]. To our knowledge, this is the first study to evaluate the activity of rifampicin, in combination with tigecycline, or daptomycin against proteinous biofilm formed by $S$. haemolyticus. In this study, we demonstrate that the tigecycline/rifampicin combination exhibits excellent in vitro activity against $S$. haemolyticus biofilm. All isolates, except two, formed a biofilm that exhibited susceptibility (defined according to EUCAST planktonic susceptibility breakpoints) to tigecycline. Therefore, we think that rifampicin plus tigecycline is a promising treatment option for biofilm-associated MRSH (methicilin-resistant $S$. haemolyticus) infections. Observation of an experimental rabbit model indicated that the tigecycline/ rifampicin combination yielded complete clearance of MRSA infections within 3 weeks [27]. In another study by Raad et al. [19], the rifampicin/tigecycline was very effective in eliminating MRSA from a silicone disc biofilm model. 
According to our studies, the combination of tigecycline/rifampicin was significantly more effective against bacteria within proteinous biofilm, than daptomycin/rifampicin. Biofilms formed by the 12 isolates of $S$. haemolyticus strains were at least three-fold more susceptible to tigecycline/rifampicin, than daptomycin/rifampicin. Nonetheless, in the case of three isolates, tigecycline/rifampicin displayed a similar effect on biofilms to daptomycin/rifampicin. Several studies have demonstrated that the effects of different combination of antimicrobial agents were strain-dependent, and for this reason, each isolate requires testing for the specific combination of the antibiotics considered for treatment $[4,23]$. We think that the daptomycin/rifampicin combination can be an alternative in treating biofilm-associated infections, caused by $S$. haemolyticus strains. These combinations inhibited $67 \%$ of biofilms, at the susceptible breakpoint of $\leq 1 \mu \mathrm{l} / \mathrm{mg}$. Lefebvre et al. [11] showed, in a rabbit model, that daptomycin/rifampicin was able to successfully reduce bone infections caused by MRSA. Similarly, John et al. [8] demonstrated the successful treatment of guinea pig MRSA foreign-body infections with daptomycin/rifampicin. This combination therapy was significantly better than daptomycin monotherapy, in an experimental, foreign-body infections model. However, Miro et al. [14] showed that daptomycin monotherapy is more effective in the treatment of MRSA endocarditis, than daptomycin combined with rifampicin.

In conclusion, this study indicates that biofilm formation is a common phenotype among S. haemolyticus strains isolated from bloodstream infections. The tigecycline/rifampicin combination was more effective against ica-independent biofilm formed by $S$. haemolyticus strains than daptomycin/rifampicin.

Open Access This article is distributed under the terms of the Creative Commons Attribution 4.0 International License (http://creativecommons.org/licenses/by/4.0/), which permits unrestricted use, distribution, and reproduction in any medium, provided you give appropriate credit to the original author(s) and the source, provide a link to the Creative Commons license, and indicate if changes were made.

\section{References}

1. Biavasco F, Vignaroli C, Varaldo PE (2000) Glycopeptide resistance in coagulase-negative staphylococci. Eur J Clin Microbiol Infect Dis 19:403-417

2. Chokr A, Watier D, Eleaume H, Pangon B, Ghnassia J-C, Mack D, Jabbouri S (2006) Correlation between biofilm formation and production of polysaccharide intercellular adhesin in clinical isolates of coagulase-negative staphylococci. Int J Med Microbiol 296:381-388

3. Entenza JM, Moreillon P (2009) Tigecycline in combination with other antimicrobials: a review of in vitro, animal and case report studies. Int J Antimicrob Agents 34:8.e1-9
4. Forrest GF, Tamura K (2010) Rifampin combination therapy for nonmycobacterial infections. Clin Microbiol Rev 23:14-34

5. Falcone M, Campanile F, Ganella M, Borbone S, Stefani S, Venditti M (2007) Staphylococcus haemolyticus endocarditis clinical and microbiologic analysis of 4 cases. Diagn Microbiol Infect Dis 57:325-331

6. Fredheim EGA, Klingenberg C, Rodhe H, Frankenberger S, Gaustad P, Fllaegstad T, Sollid JE (2009) Biofilm formation by Staphylococcus haemolyticus. J Clin Microbiol 47:1172-1180

7. Geha DJ, Uhl JR, Gustaferro CA, Persing DH (1994) Multiplex PCR for identification of methicillin-resistant staphylococci in the clinical laboratory. J Clin Microbiol 32:1768-1772

8. John AK, Baldoni D, Haschke M (2009) Efficacy of daptomycin in implant-associated infection due to methicillin-resistant Staphylococcus aureus: importance of combination with rifampin. Antimicrob Agents Chemother 53:2719-2724

9. Kristóf K, Kocsis E, Szabó D, Kardos S, Cser V, Nagy K, Hermann P, Rozgonyi F (2011) Significance of methicillin-teicoplanin resistant Staphylococcus haemolyticus in bloodstream infections in patients of the Semmelweis University hospitals in Hungary. Eur J Clin Microbiol Infect Dis 30:691-699

10. Krzymińska S, Szczuka E, Kaznowski A (2012) Staphylococcus haemolyticus strains target mitochondria and induce caspase-dependent apoptosis of macrophages. Antonie Van Leeuwenhoek 102:611-620

11. Lefebvre M, Jacqueline C, Amador G, Le Mabecque V, Miegeville A, Potel G, Caillon J, Asseray N (2010) Efficacy of daptomycin combined with rifampicin for the treatment of experimental meticillin-resistant Staphylococcus aureus (MRSA) acute osteomyelitis. Int J Antimicrob Agents 36:542-544

12. Leite B, Gomes F, Teixeira P, Souza C, Pizzolitto E, Oliveira R (2011) In vitro activity of daptomycin, linezolid and rifampicin on Staphylococcus epidermidis biofilms. Curr Microbiol 63:313-317

13. Mack D, Rohde H, Harris LG, Davies AP, Horstkotte MA, Knobloch JK-M (2006) Biofilm formation in medical device-related infection. Int J Artif Organs 29:343-359

14. Miró JM, García-de-la-Mária C, Armero Y et al (2009) The addition of gentamicin or rifampin does not enhance the effectiveness of daptomycin in the treatment of experimental endocarditis due to methicillinresistant Staphylococcus aureus. Antimicrob Agents Chemother 2009(53):4172-4177

15. Moskowitz SM, Foster JM, Emerson J, Burns JL (2004) Clinically feasible biofilm susceptibility assay for isolates of Pseudomonas aeruginosa from patients with cystic fibrosis. J Clin Microbiol 42:1915-1922

16. Otto M (2004) Virulence factors of coagulase-negative staphylococci. Front Biosci 9:841-863

17. Petersen PJ, Labthavikul P, Jones CH, Bradford PA (2006) In vitro antibacterial activities of tigecycline in combination with other antimicrobial agents determined by chequerboard and timekill kinetic analysis. J Antimicrob Chemother 57:573-576

18. Qin Z, Yang X, Yang L, Jiang J, Qu Y, Molin S, Qu D (2007) Formation and properties of in vitro biofilms of ica-negative Staphylococcus epidermidis clinical isolates. J Med Microbiol 56:83-93

19. Raad I, Hanna H, Jiang Y, Dvorak T, Reitzel R, Chaiban G, Sherertz R, Hachem R (2007) Comparative activities of daptomycin, linezolid, and tigecycline against catheter-related methicillin-resistant Staphylococcus bacteremic isolates embedded in biofilm. Antimicrob Agents Chemother 51:1656-1660

20. Rodríguez-Aranda A, Daskalaki M, Villar J, Sanz F, Otero JR, Fernando Chaves F (2009) Nosocomial spread of linezolid-resistant Staphylococcus haemolyticus infections in an intensive care unit. Diagn Microbiol Infect Dis 63:398-402 
21. Rodhe H, Mack D, Christner M, Burdelski Ch, Franke G, Knobloch JK-M (2006) Pathogenesis of staphylococcal device-related infections: from basic science to new diagnostic, therapeutic and prophylactic approaches. Rev Med Microbiol 17:45-54

22. Rodhe H, Burandt EC, Siemssen N, Frommelt L, Burdelski Ch, Wurster S, Scherpe S, Davies AP, Harris LG, Horstkotte MA, Knobloch JK-M, Ragunath Ch, Kaplan JB, Mack D (2007) Polysaccharide intercellular adhesion or protein factors in biofilm accumulation of Staphylococcus epidermidis and Staphylococcus aureus isolated from prosthetic hip and joint infections. Biomaterials 28:1711-1720

23. Steenbergen JN, Mohr JF, Thorne GM (2009) Effects of daptomycin in combination with other antimicrobial agents: a review of in vitro and animal model studies. J Antimicrob Chemother 64:1130-1138

24. Szczuka E, Kaznowski A (2014) Antimicrobial activity of tigecycline alone or in combination with rifampicin against
Staphylococcus epidermidis in biofilm. Folia Microbiol 59(4):283-288

25. Szczuka E, Urbańska K, Pietryka M, Kaznowski A (2013) Biofilm density and detection of biofilm-producing genes in methicillin-resistant Staphylococcus aureus strains. Folia Microbiol 58(1):47-52

26. Tabe Y, Nakamura A, Igari J (2001) Glycopeptide susceptibility profiles of nosocomial multiresistant Staphylococcus haemolyticus isolates. J Infect Chemother 7:142-147

27. Yin L-Y, Lazzarini L, Li F, Stevens CM, Calhoun JH (2005) Comparative evaluation of tigecycline and vancomycin, with and without rifampicin, in the treatment of methicillinresistant Staphylococcus aureus experimental osteomyelitis in a rabbit model. J Antimicrob Chemother 55:995-1002 